\title{
STD Testing and Compliance with CDC and USPSTF HIV Testing Guidelines in a Regional Cohort of Emergency Departments
}

\author{
Fredric M. Hustey, Michael P. Phelan*, Sharon O’keefe and Tracy M. Barbour \\ Emergency Services Institute, Cleveland Clinic, Cleveland, OH, USA
}

\section{Objective}

To assess compliance with CDC and USPSTF guidelines for HIV testing in a regional cohort of emergency departments.

\section{Introduction}

In 2003, the Centers for Disease Control and Prevention (CDC) in Atlanta, Georgia estimated that approximately 1 million people in the United States were living with HIV/AIDS, and that approximately $25 \%$ these were undiagnosed and unaware of their HIV infection. For many such patients the emergency department may be the only part of the health care system that is utilized. In 2006, the CDC revised their recommendations for HIV testing in a variety of care settings including the emergency department (ED). Early identification and treatment improves survival for patients with HIV. Earlier testing programs including those provided in the ED may lead to earlier detection and further reduction in the transmission of HIV in the United States. $(1,2,3,4,5)$ High risk patients should recieve screening for HIV and those patients who have a lab test for GC/chlamydia represent a high risk patient popualtion.(4) While the ED is a frequent health care access point for patients seeking evaluation for sexually transmitted diseases, ED providers may not be following guidlines for HIV testing in high risk patients.

\section{Methods}

Health system financial database review of tests billed for GC, Chlamydia, HIV and syphilis, performed in a cohort of 13 health system emergency departments (EDs). The 13 EDs included a mixture of urban and suburban hospitals, one of which was a major tertiary care center with affiliated ED residency program. Financial database was queried for all GC/Chlamydia, HIV and syphilis testing performed in the 13 health system emergency departments for a 12 month period between December 2011 and December 2012 by a trained analyst blinded to the purposes of the study. Results were then entered into an electronic database for analysis. All occurrences of GC and or chlamydia testing were then identified and prevalence of HIV and/ or syphilis testing in this population were analyzed. Proportions with $95 \%$ confidence intervals are reported.

\section{Results}

There were 13927 total occurrences of ED GC and/or chlamydia testing done in the 16 health system EDs. Three hundred ninety seven of 13927 (2.85\%; 95\%CI, 2.57\%-3.13\%) also received HIV testing. Two hundred ninety of 13927 (2.1\%; 95\%CI, 1.86-2.34\%) received HIV testing without syphyllis screen and nine hundred and eightythree of 13927 (7.1\%; 95\%CI, 6.67-7.53\%) received syphilis testing without HIV screen. Only $0.8 \%$ of patients (107/13927; 95\% CI, .65$.95 \%)$ received both HIV and syphilis screening as per USPSTF and CDC guidelines.

\section{Conclusions}

In spite of CDC and USPSTF recommendations for HIV screening in patients undergoing evaluation for STDs, only a very small proportion of ED patients meet these guidelines. In addition, ED physicians are more likely to order syphilis testing than HIV screening in this population. Further studies exploring barriers to HIV screening in patients undergoing STD assessment in the ED may help inform future projects aimed at increasing guideline compliance.

\section{Keywords}

HIV; emergency department; screening; syphilis; std

\section{References}

1. Glynn M, Rhodes P. Estimated HIV prevalence in the United States at the end of 2003. National HIV Prevention Conference; June 2005; Atlanta. Abstract T1-B1101. Available at: http://www.aegis.com/conferences/NHIVPC/2005/T1-B1101.html. Accessed February 11, 2008.

2. CDC MMWR, August 8, 2008 / 57(31);845-849 http://www.cdc.gov/ $\mathrm{mmwr} / \mathrm{preview} / \mathrm{mmwrhtml} / \mathrm{mm} 5731 \mathrm{a} 1 \mathrm{htm}$, accessed June 25, 2013

3. Branson BM. Revised recommendations for HIV testing in healthcare settings. Available at: http://www.cdc.gov/hiv/topics/testing/ resources/slidesets/pdf/

4. USPSTF Recommendations for STI Screening, http://www.uspreventiveservicestaskforce.org/uspstf08/methods/stinfections.htm

5. CDC 2010 STD Treatment Guidelines, http://www.cdc.gov/std/treatment $/ 2010$

*Michael P. Phelan

E-mail: phelanm@ccf.org 